The Java Program for Calculation of Molecular Diffusion Coefficients

\begin{tabular}{|c|c|c|}
\hline \multicolumn{2}{|c|}{ Calculation of Molecular Diffusion Coefficient } & $\square \square x$ \\
\hline \multirow{6}{*}{$\begin{array}{l}\text { File name } \\
\text { Number of diffusion data } \\
\text { Initial PFG strength (G/cm) } \\
\text { Increment of PFG (G/cm) } \\
\text { Width of PFG (ms) } \\
\text { Delay between centers of PFGs (ms }\end{array}$} & h20.txt & \\
\hline & 17 & \\
\hline & 2.187 & \\
\hline & 1.0935 & \\
\hline & 2 & \\
\hline & 100 & \\
\hline \multirow{5}{*}{$\begin{array}{l}\text { Diffusion Coefficient ( } \mathrm{cm}^{\wedge} 2 \text { is) } \\
\text { Relative Standard Deviation (RSD) } \\
\text { Amplitude } \\
\text { RSD }\end{array}$} & ter & \\
\hline & $2.28 \mathrm{E}-5$ & \\
\hline & $0.60 \%$ & \\
\hline & 102.88 & \\
\hline & $0.15 \%$ & \\
\hline
\end{tabular}

Graphic Interface

\title{
Output file:
}

File name of the diffusion data: h2o.txt Number of diffusion data: 17

Initial PFG strength $(\mathrm{G} / \mathrm{cm})$ : $\quad 2.187$

Increment of PFG $(\mathrm{G} / \mathrm{cm})$ : $\quad 1.0935$

Width of PFG (ms): 2.0

Delay between centers of PFGs (ms): 100.0

Diffusion coefficient: $\quad 2.2765056236383666 \mathrm{E}-5(\mathrm{~cm} \wedge 2 / \mathrm{s})$

Relative standard deviation (RSD): $0.6046632675963655 \%$

Amplitude: $\quad 102.88147205083558$

RSD of the amplitude: $\quad 0.14658208718889654 \%$

Total number of diffusion coefficients: 136

Positive number of diffusion coefficients: 136

Number of diffusion coefficients used: $\quad 82$

$\begin{array}{lcc}\text { PFG }(\mathrm{G} / \mathrm{cm}) & \text { Experimental data } & \text { Calculated data } \\ 2.1870 & 100.0 & 99.7448 \\ 3.2805 & 95.9639 & 95.9581 \\ 4.3740 & 90.7653 & 90.8969 \\ 5.4675 & 84.4614 & 84.7800 \\ 6.5610 & 77.5211 & 77.8600 \\ 7.6545 & 66.7511 & 70.4063 \\ 8.7480 & 60.3441 & 62.6881 \\ 9.8415 & 55.0896 & 54.9586 \\ 10.9350 & 50.3034 & 47.4419 \\ 12.0285 & 40.197 & 40.3242 \\ 13.1220 & 33.7013 & 33.7478 \\ 14.2155 & 27.8246 & 27.8101 \\ 15.3090 & 22.5337 & 22.5650 \\ 16.4025 & 18.0362 & 18.0279 \\ 17.4960 & 14.0882 & 14.1818 \\ 18.5895 & 10.9838 & 10.9848 \\ 19.6830 & 8.3637 & 8.3778\end{array}$

